\title{
Editorial
}

\section{Special Issue: Adaptive/Smart Structures and Multifunctional Materials with Application to Morphing Aircraft}

\author{
Rafic Ajaj \\ Aeronautics and Astronautics, Faculty of Engineering and the Environment, \\ University of Southampton, Highfield Campus, Tizard Building (B13), Southampton SO17 1BJ, UK; \\ E-Mail: r.ajaj@soton.ac.uk; Tel.: +44-238-059-2453
}

Received: 15 December 2014 / Accepted: 15 December 2014 / Published: 16 December 2014

Recent advances in smart structures and multifunctional materials have facilitated many novel aerospace technologies such as morphing aircraft. A morphing aircraft, bio-inspired by natural fliers, has gained a lot of interest as a potential technology to meet the ambitious goals of the Advisory Council for Aeronautics Research in Europe (ACARE) Vision 2020 and the FlightPath 2050 documents. A morphing aircraft continuously adjusts its wing geometry to enhance flight performance, control authority, and multi-mission capability.

In the last 30 years, there have been a number of international research programmes and projects on morphing wings. Many of these programmes are still active, especially in Europe. These programmes/projects have developed many adaptive/smart structures to allow large and small shape changes and they have investigated multifunctional materials to act as actuators and/or sensors. Furthermore, adaptive structures and multifunctional materials have been used to design compliant skins which are one of the main challenges of morphing wings. These skins have to be flexible in the morphing direction but rigid in other directions to maintain the aerodynamic shape of the wing and withstand the aerodynamic loads. The other main challenge facing morphing aircraft is the ability to design light weight, stiff, and robust adaptive structures that require minimal actuation power.

The use of adaptive/smart structures and multifunctional materials is not limited to morphing aircraft but has been used extensively in other fields, such as structural health monitoring, energy harvesting, suspension systems, wind-turbine blades, race cars, and many others. Therefore, we invite papers either addressing the research opportunities outlined here, or in the general topic area of adaptive/smart structures and multifunctional materials that will make a substantive contribution to the state of the art. 


\section{Conflicts of Interest}

The author declares no conflict of interest.

(C) 2014 by the authors; licensee MDPI, Basel, Switzerland. This article is an open access article distributed under the terms and conditions of the Creative Commons Attribution license (http://creativecommons.org/licenses/by/4.0/). 